\title{
Les conditions d'une gestion des compétences des cadres de l'action publique territoriale
}

Gilles Jeannot, LATTS, Ecole nationale des ponts et chaussées

(Article publié dans Politiques et management public, vol 23, n² 2, juin 2005, p. 1-19)

L'introduction du «salaire au mérite » dans quelques ministères, ou pour être plus précis d'une prime corrélée à des indicateurs de résultat ou d'activité du service, a le «mérite » de reposer le débat de la différenciation du traitement des fonctionnaires dans une visée d'efficacité (TROSA, 2004). Cette innovation, qui reproduit des pratiques du privé (DE BAYSER et alii) et d'administrations d'autres pays ${ }^{1}$ n'est cependant qu'une des voies possible qui prend le parti à la fois d'une réponse simultanée et d'une corrélation à des effets de l'action engagée. Une autre voie de transformation est aussi discutée en parallèle tant dans les entreprises que dans les administrations étrangères. Autour de la notion de «compétence », les efforts de différenciation visent plutôt à mettre en avant dans la durée le renforcement d'une capacité à faire face à des situations de plus en plus complexes qui demandent à la fois savoir-faire, capacité d'initiative et prise de responsabilité.

Après avoir précisé ce que recouvre cette orientation de gestion du personnel (1), l'article, à partir des exemples de deux catégories de cadres engagés dans l'action publique sur le territoire (les ingénieurs des travaux publics de l'Etat et les commissaires de police ${ }^{2}$ ) met en évidence l'enjeu d'une telle approche (2) et le fait que celle-ci n'est pas complètement absente des pratiques actuelles de gestion des carrières (3 et 4). Il y aurait ainsi, et souvent de manière informelle, certaines composantes d'une gestion des compétences « sans le savoir ». Enfin, à partir de la mise en relation de ces pratiques différenciées, les conditions d'un renforcement pragmatique de cette autre voie seront dégagées (5).

\section{1) La gestion des compétences : origine et définition}

\footnotetext{
${ }^{1}$ Elles ont rencontré des succès plus ou moins mitigés (OCDE, 1997; TROUSSIER, 1993 ; MARSDEN et RICHARDSON 1992).

${ }^{2}$ Les questions de carrières, comme celles de primes, font partie du noyau le plus secret des organisations publiques. Ceci explique la mobilisation de sources variées pour éclairer le sujet sous différents angles. Pour le Ministère de l'intérieur: une enquête menée auprès de commissaires de police de deux directions départementales de sécurité publique sur leur perception de leur travail et de la gestion de leur carrière $(\mathrm{N}=20)$. Cette enquête a été financée par le ministère de la fonction publique (DGAFP) dans le cadre de l'observatoire de l'emploi public et a été rendue possible par le service de modernisation et de prospective de la police nationale. Pour le Ministère de l'équipement : une enquête menée sur les compétences dans le domaine de l'eau dans trois directions départementales de l'Equipement commanditée par le Centre de formation de ce ministère à Nantes (CIFP) (N=30), grâce à Paul Kelifa, et une observation durant deux journées d'une «pré CAP », réunion informelle entre la direction du personnel et les syndicats pour préparer la commission administrative paritaire de promotion au grade d'ingénieur divisionnaire. Cela a été complété dans les deux ministères par des entretiens auprès des responsables de la gestion des carrières.
} 
L'approche par les compétences telle qu'elle est mobilisée dans les entreprises peut être définie à partir de deux spécificités. La première est de fonder une hiérarchie sur la capacité d'action efficace d'un individu dans une situation particulière. Une telle conception très proche du sens commun (est compétent celui qui réussit en situation) est aussi séduisante que problématique, il reste en effet à déterminer ce qui explique cette réussite. La seconde spécificité est alors d'intégrer dans la définition de cette capacité de l'individu autre chose que des connaissances formelles ou des savoir faire techniques. Philippe Zarifian qui a dégagé dès 1988 les éléments d'un modèle de compétence, en résume les traits : initiative (capacité à initier l'action) et responsabilité (assumer les conséquences des initiatives), ces deux éléments étant complétés d'un côté par une "intelligence pratique" qui permette de comprendre une situation singulière et pas seulement d'appliquer un savoir et d'un autre côté par une inscription dans un collectif qui seul permet l'action efficace (ZARIFIAN, 2002). Cette orientation émerge dans un contexte productif dans lequel la capacité d'initiative, de réaction à l'imprévu devient plus importante que la capacité à obéir aux prescriptions attachées à un poste.

Une telle approche, dans sa forme la plus aboutie, peut se condenser dans des grilles qui hiérarchisent des niveaux d'engagement des individus. Ainsi par exemple dans la grille d'une entreprise de formation, un cadre commercial dans sa fonction de conseil au client peut-il à un niveau élémentaire «proposer une solution technique », puis de manière plus élaborée "faciliter le choix entre plusieurs solutions techniques possibles » puis "proposer une solution stratégique (plus globale sur le moyen terme) » et au niveau le plus élevé «faciliter le choix entre plusieurs solutions stratégiques » (MASSON, PARLIER, ). Chacun de ces niveaux correspond alors pour l'entreprise évoquée un niveau différent de salaire organisé dans une grille générale pour l'entreprise.

La «logique compétence » est traditionnellement opposée au modèle des « qualifications » dans lequel des conventions collectives «qualifient» non pas le comportement de l'individu mais le poste de travail, son niveau de complexité et de technicité et indirectement le niveau scolaire qui est requis pour l'occuper. Cette dernière orientation a l'avantage de requérir un jugement sur le poste détaché de l'appréciation portée par la hiérarchie sur la personne qui l'occupe. Les réformes dites des «qualifications » à La Poste et à France télécom (Alter, 2000) s'inscrivent dans cette perspective. Tous les postes ont été hiérarchisés et le passage à un poste de niveau supérieur suppose un examen d'aptitude.

Cependant, l'approche par les compétences est à la fois plus ancienne, plus diffuse et pour l'instant plus limitée (IRIBARNE d', 2001) que ne le suggère l'image de nouveau prêt-à-porter de la GRH des grilles de compétences évoquées. Ancien, puisque comme le rappelle Jean Saglio (1987), dès 1974 un accord dans la métallurgie intègre comme critère d'évaluation l'autonomie et la responsabilité $e^{3}$. Cette innovation s'est depuis progressivement diffusée dans de nombreuses conventions collectives. Ces "grilles à critères classants" instillent donc une orientation "compétence" dans un cadre de base "qualification". Diffus en ce qu'il ne concerne pas que la gestion interne des entreprises. La validation des acquis de l'expérience (VAE), en particulier,

\footnotetext{
${ }^{3}$ Et pour ce qui concerne les expériences proprement dite de gestion des compétences, la première grille de salaire conforme à cette norme, celle de Sollac à Dunkerque, date de 1990 (CHATZIS et al. 1995).
} 
récemment reconnue comme un droit par la loi s'inscrit dans une telle perspective ${ }^{4}$; ce sont en effet bien des savoirs situés que l'on cherche à reconnaître pour les durcir dans un statut scolaire. Mais aussi limité : les entreprises qui auraient effectivement recomposé leur gestion du personnel autour des compétences demeurent l'exception ${ }^{5}$.

En outre, avant même que ce modèle ne soit vraiment mis en oeuvre, de nombreux auteurs ont déjà souligné tous les risques d'isolement du salarié face à la direction qui évalue sa compétence lorsqu'il est au sein de l'entreprise et de non transférabilité de la reconnaissance de la compétence s'il doit changer d'entreprise (ROZENBLATT, 2000). Ce dont ne disconviennent pas les promoteurs de ce modèle qui s'efforcent de proposer des règles permettant de circonvenir ces effets négatifs (PARADEISE, LICHTENBERGER, 2001). Le modèle des compétences reste ainsi plus une orientation de la gestion du personnel qu'une technique prête à l'emploi.

Parallèlement, la notion de «competency » s'impose pour les «managers » de haut niveau dans les cercles internationaux concernés par la gestion publique (HOOD, 2004 ; HORTON, 2000). Le terme "competency" ne va pas sans évoquer celui de "compétence" qui focalise aujourd'hui le débat sur la gestion du personnel dans les entreprises françaises et certains partis pris sont convergents. Une fois encore le cas britannique offre une expérience bien tranchée à travers un référentiel de compétences pour le Senior civil service qui rassemble les 3000 hautsfonctionnaires répartis dans les administrations et agences publiques. Le rapport sur la réforme de la fonction publique de Richard Wilson a proposé en 1999 une modification du système de rétribution qui inclut un référentiel, lequel n'a toutefois pas encore été mis en œuvre (FARNHAM, HORTON, 2002). Le nouveau référentiel de compétence est conçu comme un instrument de réforme culturelle et organisationnelle. Un cabinet privé, composé d'anciens fonctionnaires, a été retenu pour définir ce référentiel intitulé Leadership for results. Il se réduit à six compétences clés correspondant à des attitudes vis à vis des collaborateurs : 1) donner un but et une direction, créer une vision du futur ; 2) créer un impact personnel, montrer la voie; 3 ) penser de manière stratégique ; 4) amener chacun à donner le meilleur de soi ; 5) apprendre et améliorer ; 6) se focaliser sur les résultats et organiser la prestation de service jusqu'aux destinataires. Pour rendre la chose plus tangible, le document donne une liste de comportements qui révèlent la possession de la compétence et, plus original, d'attitudes qui trahissent le manque de la compétence : ainsi par exemple "prendre un point de vue différent pour une attaque personnelle» pour la seconde compétence, «travailler seulement avec les personnes les plus compétentes » pour la troisième, ou «être incapable de voir les choses dans la perspective d'autrui » pour la cinquième ${ }^{6}$.

D'autres pays européens, en particulier l'Allemagne et les Pays Bas, ont développé des référentiels comparables avec toutefois certaines variations. Ces référentiels accordent d'abord une place à la dimension technique de la compétence. Pour le cas de l'Allemagne, la codification de compétences techniques et juridiques s'inscrit dans une tradition datant des années 1970 (HOOD, LODGE, 2004). Les référentiels de ces deux pays mettent aussi en avant les valeurs de

\footnotetext{
${ }^{4}$ Chapitre 4 (art 137 à 146) de la loi du 17 janvier 2002 dite de «modernisation sociale ». La question d'une reconnaissance des compétences a aussi été évoquée pour l'intégration des emplois jeunes de la fonction publique territoriale (CNFPT, 2001).

${ }^{5}$ Seulement 7,7 \% des entreprises seraient concernées (COLIN, GRASSER, 2003).

${ }^{6} \mathrm{http}: / /$ www.courtservice.gov.uk/cms/3197.htm
} 
service public attendues des responsables administratifs. Ainsi aux Pays bas on évoque les notions de "conscience de l'environnement", "conscience politique", "intégrité" et "dévouement" ("dedication") (VAN VULPEN, MOESKER, 2002). Et en Allemagne des grilles évoquent des compétences attendues pour une meilleure «gouvernance» et pour des négociations ouvertes avec les « citoyens »: « capacité à établir des réseaux avec différents individus et organisations », « sensibilité aux questions d'égalité (genre, ethnique âge, handicap) » « capacité à faire confiance à des parties prenantes extérieures » (LOFFLER et alli, 2002). Ces grilles demeurent toutefois très psychologisantes et sont plus centrées sur des attitudes que sur une capacité à accomplir certaines tâches. Comme le remarque Christopher Hood (HOOD, LODGE, 2004), de telles pratiques ne permettent pas de contrer les «défaillances de système» générées par la bureaucratie, ni d'appréhender la compétence d'avenir des fonctionnaires qui se construit autour d'une capacité de gestion des interfaces ${ }^{7}$. C'est justement l'enjeu qui paraît essentiel pour l'action publique lorsqu'elle est projetée sur le territoire.

\section{2) L'enjeu d'une prise en compte des compétences dans l'action publique territoriale}

Les notions de prise d'initiative et de responsabilité, mises en avant par les démarches de compétence apparaissent déterminantes pour l'efficacité de l'action publique territoriale. Si on suit la synthèse de Duran et Thoenig (DURAN, THOENIG, 1996), l'action publique, toujours partagée entre de multiples intervenants, se construit autour de la résolution de problèmes ou de la mobilisation d'acteurs du développement. Les problèmes sociaux, les risques et les questions de sécurité, l'environnement, ou les dynamiques économiques résistent à toute répartition des compétences ou codification des interventions. Même si l'action partagée est toujours encadrée par diverses procédures ou dispositifs, de nombreuses observations mettent en évidence le fait que ces cadres restent sans prise sur l'action s'ils sont simplement appliqués de manière formelle et routinière (JEANNOT, 2005). En d'autres termes, même si elle se coule dans des procédures de plus en plus nombreuses, l'action ne peut émerger que si les procédures et les ressources de chaque organisation sont saisies de manière volontaire, selon un projet cohérent et convaincant à la fois pour l'organisme public considéré et pour ses partenaires. S'il y a bien une construction institutionnelle de l'action publique sur le territoire, elle ne s'actualise pas sans l'initiative responsable d'agents publics de l'Etat et des collectivités territoriales et sans leur intelligence pratique de ces situations complexes. Illustrons ceci à partir de deux enquêtes l'une concernant l'activité d'ingénieurs du ministère de l'Equipement concernés par la question de la pollution des rivières, l'autre celle des commissaires de sécurité publique.

L'exemple de l'application de la loi sur l'eau met en évidence le caractère insuffisant d'une intégration purement institutionnelle de l'action. La protection des rivières contre les pollutions est encadrée par la loi sur l'eau de 1992. Cette loi organise l'usage d'instruments comme les «plans de zonage » qui fixent au niveau de la commune les limites de l'assainissement individuel et collectif ou les SAGE (Schémas d'aménagement et de gestion de l'eau) qui définissent au

\footnotetext{
7 « The capacity to operate across diverse systems, within, across and beyond government (HOOD, LODGE, 2004, p. 328). L'importance de la «productivité des interfaces» a été soulignée par Pierre Veltz pour le secteur privé «L'efficacité ne dépend plus de l'intensité du travail programmé de chacun, mais de ce qui se passe entre les individus et les groupes au travail, et qui échappe en partie à toute programmation. L'organisation -entendue comme la qualité de la coopération et des interfaces entre acteurs d'une chaîne productive- devient le facteur de performance central » (VELTZ, 2000, p. 17). Elle trouve aussi de nombreuses équivalences dans le secteur public (JEANNOT,1997).
} 
niveau de chaque bassin les objectifs de propreté de l'eau. A cette loi correspond une organisation interne des administrations: pour le Ministère de l'Equipement la séparation progressive entre un service chargé de la maîtrise d'œuvre des équipement d'assainissement pour le compte des communes (tuyaux de collecteurs d'égouts, stations d'épuration) et un service plus juridique en charge de l'application de la loi. En outre dans de nombreux départements, s'ajoute une mission interservices de l'eau qui permet de mettre en relation les différents ministères concernés. Le traitement des eaux de surface est ainsi l'objet d'une des formes d'intégration institutionnelle les plus abouties de l'action publique territoriale. Et pourtant, l'enquête qui a porté sur trois directions départementales de l'Equipement a montré que cette intégration institutionnelle ne donne tous ses résultats que s'il elle est accompagnée d'une intégration professionnelle. Ainsi, par exemple, si la loi prévoit les mêmes procédures en tout lieu, les risques de pollution dommageables sont différenciés sur le territoire. Certains sites (par exemple un lac ou un canal désaffecté) sont particulièrement sensibles aux rejets et nécessitent une attention particulière. Il faut donc à la fois connaître les outils des sciences de l'environnement pour identifier ces sites et comprendre les facteurs d'une possible dégradation et il faut maitriser les instruments juridiques répressifs pour empêcher ces dégradations. Le contraste entre les trois Directions départementales étudiées est ici frappant. La première ne dispose que des outils de diagnostic. Un technicien collecte et traite des données sur des sujets très sophistiqués comme les mouvements des effluents en milieu marin, mais ses données sont peu mobilisées par les services chargés de la résorption des pollutions. A l'opposé dans un autre département, le responsable du service a une bonne connaissance du cadre juridique mais son service ne dispose pas d'informations précises mettant en évidence les principaux enjeux. Entre les deux, les services de la troisième direction départementale maîtrisent les deux domaines autour d'un ingénieur des travaux public chef de la cellule et d'une chargée d'étude contractuelle spécialisée en environnement. Cela permet en particulier de passer à une politique répressive organisée en engageant des actions de verbalisation ciblées et en s'entendant avec le procureur de la république pour que les procès verbaux établis ne restent pas sans suite. L'action efficace naît ainsi de la capacité à mobiliser à bon escient les multiples ressources offertes par le cadre juridique.

Dans l'enquête menée auprès des commissaires de sécurité publique, ceux-ci mettent, de même, en avant de telles compétences composites situées à la jonction de plusieurs domaines. La question du traitement du caractère événementiel de l'activité de police, revient ainsi par exemple régulièrement dans les entretiens. Le travail policier est fondamentalement réactif, il est construit autour de l'événement et de diverses sollicitations qui génèrent et contraignent à la fois l'action. Cela pose tout d'abord la question du respect des procédures, garanties de libertés publiques, dans une situation d'urgence. Pour cela la maîtrise du droit est un prérequis. Elle suppose en outre d'être constamment actualisée. Mais cela ne suffit pas, il faut aussi une capacité pédagogique pour faire passer les réformes auprès du personnel et une capacité à juger en action pour que, dans le feu de l'action, les procédures ne soient pas oubliées. Cela pose ensuite la question de la rationalisation des interventions. Le risque est grand de subir continuellement les sollicitations et de ne jamais sortir d'une pratique artisanale. Les commissaires rencontrés évoquent ainsi la nécessité de savoir émerger de l'événement. Une compétence particulière consiste alors à savoir, malgré tout, générer des formes de rationalisation des interventions qui optimisent la capacité de réponse aux événements. Cela suppose à la fois de pouvoir gérer l'organisation en interne et de savoir tenir sa place entre le préfet, le procureur et le maire pour faire reconnaître ce qui relève des prérogatives de la police. 
Dans ces deux exemples, les compétences évoquées sont à la fois collectives et individuelles. Dans le cas des services de police de l'eau, par exemple, ce ne sont pas les mêmes personnes qui maîtrisent dans le détail les savoirs scientifiques et juridiques, mais leur coordination suppose la capacité d'intégration du chef de bureau. Dans ces deux cas, de même, l'action efficace suppose la maîtrise de techniques (scientifiques ou juridiques) et ne découle pas seulement des comportements auxquels se réfèrent exclusivement certaines grilles de gestion des compétences de fonctions publiques d'autres pays. Les connaissances techniques et juridiques et la manière de s'en servir ne peuvent être négligées; les dimensions «managériale et «technique» de la compétence sont profondément intriquées.

Les mêmes enquêtes sur les ministères de l'Equipement et de l'Intérieur mettent aussi en évidence les effets négatifs d'une non reconnaissance de ces compétences. D'un côté, si la prise d'initiative et de responsabilité est plus motivée par la satisfaction de voir les conséquences de son intervention, que par un retour financier immédiat, la stagnation dans la carrière génère une démotivation. De l'autre, ne pas promouvoir les agents qui ont fait preuve d'une capacité à prendre en charge l'action publique dans ses différentes dimensions, c'est se priver à un niveau supérieur de cadres ayant les capacités pour une approche à la fois pragmatique et globale.

\section{3) La gestion de carrière des ingénieurs des travaux publics de l'Etat}

Le corps des ingénieurs des travaux publics de l'Etat offre un exemple tout à fait intéressant pour illustrer le fait que le secteur public peut pratiquer la gestion des compétences "sans le savoir", mais aussi pour mettre en évidence les souplesses (PALLEZ,1999) incluses dans le statut de la fonction publique.

Le ministère de l'Equipement comprend deux corps d'ingénieurs l'un issu pour l'essentiel en recrutement initial de l'école polytechnique (ingénieurs des ponts et chaussées ${ }^{8}$ ) et l'autre qui lui est subalterne, le corps des ingénieurs des travaux publics de l'Etat (ITPE). Ces deux corps ont permis de remplir la plupart des fonctions techniques et de management des services de ce ministère qui, dans les départements, ont la charge des routes et de l'urbanisme. Si, à l'origine, à ces deux corps correspondaient des attributions de fonctions hiérarchisées et distinctes, progressivement les postes de direction ont été ouverts aux ingénieurs originaires du corps des travaux publics de l'Etat.

Dans cette situation de concurrence, le syndicat de défense des intérêts du corps des ITPE (SNITPECT) a privilégié une stratégie orientée vers l'expansion du corps plutôt qu'une logique de gestion interne à l'ancienneté. L'objectif premier était de permettre à des membres du corps d'occuper les fonctions de directeur départemental de l'Equipement. Ceci se traduit par un choix de co-gestion (avec la direction du personnel du ministère de l'Equipement), les deux acteurs s'entendant pour promouvoir les ingénieurs qui ont le «potentiel » d'occuper de telles fonctions indépendamment de toute logique d'ancienneté. La stratégie a été payante puisqu'en 2002 plus de

\footnotetext{
${ }^{8}$ Ce corps a récemment été fusionné avec d'autres corps formés d'ingénieurs issus de la même école, ingénieurs géographes, ingénieurs de l'aviation civile et ingénieurs de la météorologie ( décret 2002-523 du 16 avril 2002 portant statut particulier des ingénieurs des ponts et chaussées. Une fusion comparable des corps d'ingénieurs de travaux (dont les ITPE) est en cours de discussion.
} 
la moitié des directions départementales de l'Equipement sont occupées par des ingénieurs issus de l'école nationale des travaux publics de l'Etat'.

\section{Les règles de gestion}

La sélection pour la promotion des ingénieurs des travaux publics est très marquée. Le corps des ITPE comprend deux niveaux de grade.Sur 3300 membres du corps en position normal d'activité seuls $45 \%$ accèderont au deuxième niveau «divisionnaire » et au second niveau de fonction ${ }^{10}$ et $5 \%$ deviendront ingénieurs des ponts et chaussées par concours interne ${ }^{11}$. Le changement de grade, outre la différence de salaire renforcée par une forte hiérarchisation des primes, permet aussi de voir évoluer notablement les responsabilités confiées. Les postes ouverts au ministère de l'Equipement à ces ingénieurs sont hiérarchisés en trois niveaux. Les postes de niveau 1 (par exemple, responsable d'une subdivision territoriale généraliste sur quelques cantons, ou chargé d'études) sont ouverts aux ingénieurs débutant dans la carrière, les postes de niveau 2 (ex. chef de service routier ou logement dans un département) et de niveau 3 (ex : directeur départemental) sont ouverts aux ingénieurs divisionnaires ${ }^{12}$.

Le passage au divisionnariat se décide dans une CAP de promotion. Le changement de grade est effectif au moment où l'ingénieur promu prend son premier poste de niveau 2. Cette CAP est précédée d'une "pré-CAP" » qui rassemble direction du personnel et syndicats et dans laquelle se joue en grand part la décision.

\section{La formation d'un jugement partagé}

L'observation des discussions lors de la «pré-CAP » révèle en premier lieu un certain effort pour objectiver le jugement porté sur les individus à travers une procédure stricte de présentation des dossiers. Pour chaque postulant, une secrétaire lit l'ensemble des intitulés des postes occupés et des annotations rédigées par les supérieurs hiérarchiques (les notes chiffrées ne sont jamais évoquées) ${ }^{13}$. Ces données sont alors l'objet d'une interprétation collective. Certains éléments relèvent d'un code connu de tous ${ }^{14}$, comme le fait que l'annotation «bon ingénieur » soit comprise dans un contexte où les annotations sont volontiers laudatrices comme «élément

\footnotetext{
${ }^{9}$ Les ingénieurs des ponts issus de polytechnique occupent $33 \%$ des directions départementales (mais encore $95 \%$ des directions régionales), $42 \%$ sont occupées par des ingénieurs des travaux publics de l'Etat devenus ingénieurs des ponts par le concours interne, et $12 \%$ par des ingénieurs des travaux publics de l'Etat (CHATZIS, RIBEILL, 2004, p. 32).

${ }^{10}$ Evaluation fournie par le SNITPECT, il y a en fait trois moments de promotion, le principal entre 34 et 54 ans permet aux ingénieurs divisionnaires d'occuper les fonctions de second niveau d'une part et une promotion «principalat » après 55ans et une promotion 6mois avant la retraite d'autre part, qui offrent les avantages statutaires mais pas l'accès au deuxième niveau de fonction. Au final au moment de la retraite l'ensemble des ingénieur sera promu.

${ }^{11}$ Cette voie de promotion ne sera pas abordée ici. Ont peut cependant remarquer que les épreuves du concours sont principalement construites autour d'une mise en situation (ex rédaction d'une lettre de propositions d'action au Préfet à partir d'un volumineux dossier sur une opération routière ou d'aménagement) qui n'est pas étrangère à la prise en compte des compétences.

12135 ingénieurs des TPE occupent ce niveau de fonction auquel ne correspond pas un grade mais une surindiciation.

${ }^{13}$ Cet exercice suppose une technologie bureaucratique non négligeable pour collecter toutes les appréciations, même lorsque les ingénieurs partent en détachement,les conserver au fil des années et les rassembler sur de petites fiches de bristol maniables.

${ }^{14}$ La presse syndicale a pu même sur une mode humoristique présenter des grilles de déchiffrage de ces annotations.
} 
médiocre ». Des discussions peuvent aussi s'engager pour interpréter le sens de la formule de tel ou tel notateur connu ou pour évaluer le niveau de complexité effectif d'un poste occupé, ceci en particulier pour les agents en détachement dans les collectivités locales, sur des postes moins connus de tous. Mais le croisement de l'avis donné par au moins trois ou quatre notateurs d'une part, et la liste des postes occupés d'autre part, donnent des éléments de jugement assez solides au moins pour les cas extrêmes (l'ingénieur un peu rigide qui a rencontré des problèmes de management d'une part ou l'ingénieur très brillant qui a épaté tous ses notateurs dans des postes difficiles, par exemple) ${ }^{15}$.

En second lieu, cette observation, en situation, des commissions de promotion montre une convergence de la direction et des syndicats autour d'un principe central de compétence au management. Si la direction peut afficher officiellement des critères de soumission à l'intérêt général (accepter une mobilité géographique pour occuper des postes dans l'Est ou le Nord du pays plutôt que dans les zones plus ensoleillées), et les syndicats des valeurs d'égalité de traitement, ils convergent dans les choix effectifs sur la valorisation de critères perçus comme caractéristiques des positions de management. C'est en premier lieu la pluridisciplinarité (s'occuper de routes mais aussi d'urbanisme) qui permettra à terme de diriger une DDE. C'est aussi le primat des fonctions de management du personnel sur les fonctions techniques. Cela s'apprécie à travers le nombre d'agents sous la responsabilité hiérarchique de l'ingénieur considéré et aussi à travers l'obligation -hors cursus de spécialistes- d'avoir occupé un "poste à risque » (subdivisions territoriales et «parc» de matériel offrant des prestation d'entretien routier), le risque étant de conflits possible avec des élus ou avec le personnel fortement syndicalisé dans certains services

De manière plus fine, le traitement statistique d'un document produit en séance -le codage par un représentant du syndicat minoritaire des critères de promotion des ingénieurs- met en évidence le poids des critères comportementaux. Le représentant de ce syndicat minoritaire, dans une visée d'objectivation, remplit pour lui-même une liste de critères pour chaque individu. Il y a d'abord des critères objectifs partagés (la mobilité géographique, la mobilité professionnelle, l'occupation d'un poste « à risque ») qui offrent peu prise à l'interprétation et qui sont d'ailleurs affichés par la direction du personnel comme des conditions de la promotion dans les parcours standards. Il y a aussi des critères sur la capacité à gérer les subordonnés, la hauteur de vue et la capacité d'innovation. Ces derniers sont appréciés de manière plus subjective, mais le codage reflète bien la tonalité globale de la discussion. L'analyse statistique des corrélations entre ces critères et la décision de promotion montre alors, de manière remarquable, que les seules relations significatives concernent ces critères subjectifs et la capacité d'initiative des individus. Cela renforce l'impression de la volonté partagée de privilégier les ingénieurs ayant « le potentiel » de faire des carrières jusqu' au plus haut niveau.

Au total, à travers les données objectives et subjectives se dessine la reconnaissance d'un profil de compétence de manager. L'occupation d'un poste opérationnel de premier niveau garantit un minimum de capacités relationnelles vis-à-vis des élus mais aussi vis à vis des agents. La

\footnotetext{
${ }^{15}$ Cela n'empêche bien sûr pas d'autres éléments non explicites de peser comme l'activité au sein du syndicat majoritaire. Dans certains cas cependant ces arguments ne peuvent pas toujours suffire comme en témoigne la réaction d'un représentant syndical qui défend un membre actif de son syndicat et qui conscient du caractère non plaidable du cas finit en disant : «j'aurai au moins essayé ».
} 
mobilité géographique et fonctionnelle permet d'avoir une maîtrise relative de différents domaines techniques et juridiques et une sensibilité à la variabilité des situations. Enfin, derrière les critères plus subjectifs, est appréhendée une capacité à ne pas simplement mettre en œuvre, «faire tourner son service », mais aussi à faire émerger des projets nouveaux en composant des ressources et des acteurs inédits.

\section{L'enjeu des compétences techniques}

Aujourd'hui cependant, si les compétences de conduite d'un service demeurent importantes, elles ne suffisent pas. Le niveau technique des services du ministère est mis en cause alors que la décentralisation puis l'action de la commission européenne (application de la directive "services" aux prestations d'ingénierie publique) mettent de fait de plus en plus ces services en concurrence avec d'autres. Des compétences plus techniques s'avèrent de plus en plus nécessaires. Deux réponses successives ont été données. La première a consisté à créer des filières de spécialistes gérées à partir du jugement des pairs sur le modèle de la gestion de la recherche. Cela ne permettait cependant que de répondre au cas des ingénieurs en bureaux d'études. La seconde réponse a alors eu comme objectif de reconnaître des compétences de "généraliste de domaine", c'est à dire celles d'ingénieurs engagés dans des pratiques opérationnelles qui au fil des postes cumuleraient différents points de vue sur un même domaine. La direction du personnel a dessiné des parcours permettant l'accumulation de compétences techniques dans un domaine qui ne relève plus de la logique de spécialisation croissante propre aux experts de haut niveau ou aux chercheurs. Ainsi par exemple dans le domaine de l'assainissement, évoqué précédemment, on pourra envisager un premier poste de conduite de chantier de stations d'épuration, suivi d'un deuxième poste dans un service de conseil amont auprès des décideurs dans ce domaine, puis d'un poste dans les services juridiques chargés de l'application des lois concernant la pollution des rivières. L'ingénieur pourra alors être promu et prendre la responsabilité générale du service environnement ou travailler dans une commune au service d'assainissement. Il aura acquis dans un même domaine des compétences techniques, juridiques, d'organisation de chantier et des capacités de négociation et de conduite d'équipe ${ }^{16}$.

L'exemple du Ministère de l'équipement montre donc qu'il est possible, en accord avec les syndicats, de créer des procédures de promotion qui tiennent compte non seulement d'un titre et d'une ancienneté, mais d'une compétence. On retrouve de tels aspects dans le cas de la gestion des commissaires de police mais enchâssés dans des règles et un contexte organisationnel moins favorables.

\section{4) La gestion des carrières des commissaires de police}

Le corps des commissaires apparaît du point de vue sociologique pris en étau entre celui des officiers de police d'une part et le corps préfectoral de l'autre. Les officiers disposent d'un poids politique lié à leur masse. Ils peuvent grignoter par le bas les circonscriptions, ou, à terme, monter en légitimité à travers l'accès à la catégorie A. Le corps préfectoral dispose d'autres ressources propre à un grand corps interministériel, et tout montre que la décentralisation est loin

\footnotetext{
${ }^{16}$ Cette orientation qui a été concrétisée en 2004 par la création de dix commissions d'évaluation pour dix domaines a été contestée par le syndicat SNITPECT qui y a vu le signe d'une filièrisation du corps.
} 
de réduire son influence. Les préfets restent par exemple signataires des contrats de sécurité dans la récente loi d'orientation de sécurité intérieure.

La porosité entre corps n'est pas la même des deux côtés. Les entrées par le bas ne sont pas négligeables. Si on ajoute au concours interne, les promotions au choix (rares ces dernières années) et les "faux externes" (à savoir les officiers de police qui durant leur formation ou les premières années d'affectation passent le concours externe en ayant acquis une première expérience professionnelle de police), c'est environ la moitié des commissaires qui sont issus de l'interne. A cela, il faut ajouter les commandants fonctionnels en charge d'une circonscription qui ne sont pas membres du corps des commissaires mais qui, symboliquement aux yeux de l'extérieur, y sont fortement associés. Les sorties par le haut sont, elles, beaucoup plus limitées. Hormis quelques postes dans le privé, occupés plutôt après la retraite, un quota de postes de souspréfets et quelques positions de responsables sécurité dans des entreprises publiques ou ministères, on est loin d'un corps interministériel.

Dans ce contexte, le syndicat majoritaire s'efforce de contenir la montée des officiers en pesant pour le maintien d'épreuves scolaires permettant le passage d'officier à commissaire et fait pression pour limiter le nombre de directions de commissariats confiées à des officiers. Par ailleurs il a joué la carte d'un mimétisme avec le corps des préfets et a obtenu récemment une transcription de l'obligation de "mobilité" propre aux administrateurs civils. Dans une organisation dans laquelle, comme l'a montré Dominique Monjardet (1987), la tension entre valorisation des compétences (entendues comme expérience) ou des qualifications (entendues comme formation initiale et savoirs formels) organise les relations, le syndicat majoritaire des commissaire joue plutôt la seconde carte.

\section{Les règles de gestion des carrières}

La différenciation des carrières des commissaires est moins marquée que celle des ingénieurs des TPE mais néanmoins réelle. Il y a 3 niveaux de grade ${ }^{17}$. Le passage du premier palier, commissaire à commissaire principal, est quasiment automatique environ dix années après la sortie d'école. Le passage à divisionnaire, en revanche, est plus sélectif : après 20 années de carrière sur les 361 commissaires entrés dans la police entre 1980 et 1985, 6 sont restés commissaires, 162 commissaires principal et 193 sont passés divisionnaires, soit une bonne moitié $^{18}$.

Pour les commissaires, contrairement aux ingénieurs des TPE, la mutation précède la promotion. Il faut aller "chercher" sa promotion en postulant sur un poste de niveau plus élevé. Ce mode de gestion, dont on comprend l'intérêt pour forcer les mobilités, conduit de fait à prendre en compte la possibilité du passage au grade supérieur au coup par coup en fonction des réponses aux "télégrammes" annonçant une ouverture de poste.

\footnotetext{
${ }^{17}$ Commissaire, commissaire principal, commissaire divisionnaire : Décret $n^{\circ} 220-936$ du 14 juin 2002 modifiant le décret $\mathrm{n}^{\circ}$ 95-6555 du 9 mai 1995 portant statut particulier du corps de conception et de direction de la police nationale.

${ }^{18}$ Source : bureau de gestion des commissaires. La différenciation s'atténue progressivement avec le temps, sur les 325 agents entrés entre 1975 et 1979, un seul est encore commissaire et 76 commissaires principaux.
} 
Enfin la gestion est déléguée par la direction générale aux directions centrales fonctionnelles (sécurité publique, police judiciaire, renseignements généraux,... ) largement indépendantes. Des quotas de promouvables sont en effet attribués à celles-ci qui prennent ensuite les décisions concernant les individus. Cela conduit à un mode de gestion différencié selon la taille de ces directions (les petites directions comme les renseignements généraux connaissent l'ensemble des agents, ce qui est plus difficile en sécurité publique) et multiplie les effets de pyramidage puisque les variations selon les âges ne s'équilibrent pas entre directions.

\section{La perception des règles de gestion}

L'enquête sur la perception des carrières montre que les compétences peuvent être perçues, comme on l'a vu à propos de la gestion de l'événementiel, mais aussi reconnues. La responsable du bureau de gestion des commissaires rappelle ainsi son étonnement en début de carrière devant les discussions qui animaient la rencontre au café lorsqu'un poste se libérait : qui l'occupera ? qui occupera le nouveau poste libéré par ce transfert etc... «le plus remarquable était qu'ils se trompaient assez peu ». Derrière cette anecdote se révèle l'existence de principes partagés d'évaluation des individus. Cependant, les commissaires rencontrés s'ils ne nient pas cette culture commune, mettent aussi en avant les difficultés d'une prise en compte effective des compétences dans les carrières.

En premier lieu, la visibilité des individus n'apparaît pas égale. Contrairement au cas des ingénieurs des TPE, les règles formelles ne facilitent pas la comparaison des situations. Dans un mode de gestion où la promotion précède la mutation il est possible de discuter en un même lieu et de mettre ainsi en balance toutes les demandes de promotion. Pour les commissaires, la confrontation de dossiers se fait de manière limitée à propos d'un poste particulier avec une concurrence variable, plus liée à l'attractivité du poste qu'aux qualités des individus. Par ailleurs, la gestion par les directions fonctionnelles introduit un fort coût au changement de direction. Il faut en effet se faire connaître dans la nouvelle direction avant de se voir promu. Les commissaires rencontrés mettent alors moins en cause la capacité collective de l'institution à reconnaître et valoriser de telles compétences, que l'aléa lié au fait que, selon les cas, les individus peuvent échapper à une évaluation si des circonstances extérieures (visite d'une inspection, passage de leur supérieur hiérarchique en administration centrale, proximité d'un élu en vue...) ne les rend pas visibles. D'où le risque d'effet de "cour" : il faut non seulement agir efficacement mais aussi le faire savoir des responsables de la gestion de l'attribution des postes et pour cela se «montrer » soit dans des groupes de travail nationaux, soit auprès d'élus qui ont du poids soit dans les médias.

En second lieu, les règes de gestion ne tranchent pas clairement entre caractérisation des postes et jugement sur les individus. Le principe de promotion lié à la mobilité repose sur une qualification des niveaux de complexité des postes. C'est le cas de manière assez nette pour les officiers, cela ne l'est que dans les grandes lignes pour les commissaires. D'une part le délai peut être long entre la prise de poste de niveau supérieur et la promotion effective. D'autre part, tout cela suppose l'existence d'une «nomenclature » qui définisse les niveaux de chaque poste et les hiérarchise ${ }^{19}$.

\footnotetext{
${ }^{19}$ La variation de difficulté selon la taille de population, le taux de délits, ou la personnalité du maire concerné est plus marquée pour les commissariat que pour l'équivalent au ministère de l'Equipement et mérite une évaluation plus fine.
} 
Or celle-ci existe et est de fait utilisée, mais elle n'est pas publiée. En effet, si une telle nomenclature était lue de manière mécanique, elle bloquerait toute marge de manœuvre en forçant la promotion immédiate pour les commissaires prenant un poste de niveau supérieur. Le choix de l'entre-deux permet de laisser la porte ouverte à d'autres critères que ceux du niveau du poste occupé et concerner les qualités des personnes.

En troisième lieu, les jugements sur les individus mêlent «mérite » et « compétence ». Alors que la présentation de l'enquête met en avant le terme de "compétence", celui-ci est spontanément remplacé par celui de "mérite". Il y a là plus qu'une question de vocabulaire maison. "Mérite" peut recouvrir deux choses : l'expression d'une qualité (ce qui pourrait le relier à la compétence) et le droit légitime à une récompense (ou à une peine) ${ }^{20}$. C'est le second aspect qui est souvent visé par les personnes interviewées. C'est en particulier le cas lorsqu'un commissaire a accepté un poste peu recherché et qu'il attend en retour une reconnaissance sous forme d'un poste plus attractif ou d'une promotion. Il semble que ce retour puisse réellement exister, un bon nombre des personnes rencontrées reconnaissant que l'administration "sait se souvenir". Le passage entre "compétence" et "mérite" est si naturel pour les intéressés qu'il faut rappeler que, dans ce second sens, les deux notions sont différentes : le fait de se sacrifier en acceptant un poste dans une région peu attractive ne garantit pas que l'on soit capable d'occuper un poste de niveau supérieur.

Enfin, même lorsque le principe d'une prise en compte des compétences est partagé, il reste à savoir quelles compétences valoriser. Les commissaires de sécurité publique peuvent être considérés soit comme des «animateurs de politique publique de sécurité », largement orientés vers le partenariat avec les autres administrations, soit comme des «chefs de police » chargés de rendre opérationnelles les équipes de policiers sur le terrain. Les critères de compétences ne sont pas les mêmes dans les deux perspectives. Les commissaires rencontrés sont loin de définir leur activité en termes de politiques publiques de manière comparable à celles de directeurs d'administrations territoriales et se conçoivent d'abord comme des «chefs de police ». Mais le syndicat majoritaire, dans une stratégie de promotion tout à fait classique, a choisi de plaider pour un alignement sur le modèle des corps supérieurs: préfets ou directeurs d'administrations déconcentrées. Ce faisant cette orientation valorise plus les compétences de conduite de politique publique que de management d'équipe. Cette dualité d'orientation ne facilite pas la constitution d'une critériologie partagée de reconnaissance des compétences.

\section{5) Prendre en compte les compétences}

Si on met en perspective les deux exemples présentés ici rapidement, plusieurs éléments communs s'imposent concernant les conditions préalables à une prise en compte des compétences.

a) Le principe d'une prise en compte des compétences sur le moyen terme à travers l'attribution des postes est loin d'être contre-culturel et une observation fine des pratiques effectives de promotion montre que de tels critères sont dors et déjà partiellement mis en œuvre. Plus que d'un

\footnotetext{
${ }^{20}$ Le terme de mérite a été associé à l'atteinte d'un résultat chiffré (le taux d'élucidation des affaires) par un des commissaires. Ce cas pose la question de l'attribution d'un résultat d'une action collective à un individu en effet celui-ci se plaignait du fait que le bon résultat avait valu une reconnaissance au commissaire en charge du service concerné, et au directeur départemental mais pas vraiment à lui qui était chef de district.
} 
retour immédiat sur la productivité, celle d'une appréciation dans la durée des capacités personnelles et des volontés d'engagement dans la vie professionnelle se coulent dans des pratiques et valeurs existantes. Si certains aspects, comme le recrutement par concours sur épreuves scolaires en fin de formation initiale ${ }^{21}$ ou certaines pratiques dissociant l'évolution des grades et le parcours professionnel, sont très éloignés d'un modèle de compétence, d'autres, autour de la construction de carrières différenciées, sont plus compatibles avec un tel modèle. Une telle prise en compte des compétences ne se réduit pas à l'introduction de nouveaux "outils". Ses conditions de possibilité sont profondément corrélées aux caractéristiques globales des corps au sein desquels se déroulent les carrières. La position du corps parmi ses voisins, le pyramidage des niveaux au sein de celui-ci, les lieux et occasions de décision, les modalités pratiques de connaissance des individus jouent sur la possibilité de prendre en compte des compétences.

b) Le rôle des syndicats est bien évidemment essentiel. L'exemple des ingénieurs des travaux publics correspond de ce point de vue à un cas de figure très favorable dans lequel le syndicat a jugé qu'il était de l'intérêt collectif du groupe de contribuer à une transparence sur l'évaluation des agents en échange d'un accès aux fonctions de directeur départemental jusqu'alors réservées aux ingénieurs issus de l'école polytechnique. Le cas des commissaires de police l'est moins, le syndicat majoritaire, soucieux en particulier de contenir la poussée du corps des officiers de police, met en effet en avant des critères liés au niveau de formation juridique qui ne recouvre qu'une partie des compétences. Marcel Pochard remarque en substance que, si le statut de la fonction publique permet tout ce dont peut rêver un promoteur de méthodes moderne de gestion du personnel, il permet aussi l'émergence de syndicats corporatistes qui bloquent l'usage effectif de ces possibilités en confortant des tendances à la bureaucratisation : "Si le statut n'interdit pas (...) les formes de gestion vertueuses, il n'empêche pas non plus les autres et on peut se demander au contraire s'il ne les engendre pas peu ou prou"(CONSEIL D'ETAT, 2003, p. 263).

c) Les deux exemples, malgré leurs différences, font aussi apparaître l'importance des compétences techniques : revalorisation des savoirs techniques de domaines pour les ingénieurs, savoirs propres au droit pour les commissaires. C'est en ce sens que l'on peut comprendre l'émergence du terme de "métier" pour caractériser des tentatives en faveur d'une prise en compte des compétences ${ }^{22}$. L'orientation vers les compétences ne saurait ainsi - à la manière de la grille du Senior civil service britannique - se limiter à la promotion d'un modèle généraliste de "manager" garant de l'optimisation de la production de services. Il ne s'agit pas d'opposer à des attitudes managériales la maîtrise de savoirs constitués, mais une certaine manière de mobiliser les savoirs : des compétences composites nées au confluent du droit, de savoirs techniques et de l'expériences des personnes et des organisations. Ce sont ces compétences qui sont susceptibles de répondre aux "problèmes" partagés autour desquels se construit l'action publique territoriale.

d) La question de la détermination du niveau de compétence des individus reste bien sûr délicate. L'exemple des ingénieurs des travaux publics révèle cependant qu'il est possible, sans lever tout

\footnotetext{
21 Il ne faut pas négliger qu'il y a toujours quelque anachronisme à juger selon des critères managériaux contemporains des règles établies sur des visées très différentes, l'enjeu de l'introduction du concours étant d'abord de créer une administration indépendante du politique, le concours était alors la garantie d'un recrutement selon le «mérite » entendu en un sens différent de celui d'aujourd'hui (DREYFUS, 2000).

${ }^{22}$ Cf journée sur l'approche "métier" dans le respect d'une fonction publique de carrière (OBSERVATOIRE DE L'EMPLOI PUBLIC, 2003). On peut aussi dans cette perspective s'interroger sur la proposition du rapport du conseil d'Etat qui envisage la création de grandes fonctions qui écraseraient certaines spécificités de métier.
} 
risque, de porter un jugement raisonné sur les individus. Cela suppose à la fois l'existence d'un lieu de discussion et d'une construction bureaucratique (le dossier individuel) et sociale (la confrontation direction/ syndicats) de cette discussion ouverte. Cependant, dans ce cas, comme dans celui des commissaires, le fait de ne jamais perdre de vue une évaluation des postes occupés par les individus et une appréciation de leur niveau de difficulté (la nomenclature) apparaît comme un élément essentiel de l'objectivation du jugement. L'attitude de l'individu ou sa réussite en situation n'est jamais appréciée de manière abstraite mais toujours rapportée aux caractéristiques des postes occupés et de ceux qu'il demande d'occuper. Les deux types de jugement sur les pratiques des individus (compétences) et sur les postes (qualification) opposés dans l'introduction apparaissent en fait complémentaires ${ }^{23}$.

e) Enfin, prendre en compte les compétences et hiérarchiser les individus ne se fait pas sans prise de parti sur les missions principales à valoriser. La compétence n'existe toujours que par rapport à une forme d'efficacité visée (JEANNOT, PERALDI, 1991). Faut-il privilégier pour les ingénieurs des travaux publics de l'Etat des parcours de managers capables d'assumer une direction départementale, complétés par des spécialistes gérés comme des chercheurs ou faut-il mettre en avant des "généralistes de domaine » qui restent fondamentalement des ingénieurs accumulant et enrichissant au cours de leur carrière leurs connaissances techniques, juridiques et administratives d'un domaine particulier ? Faut-il, pour les commissaires, se donner comme modèle de référence la figure d'un "chef de police" qui à tous les niveaux reste au plus près de l'opérationnel et des hommes qu'il dirige ? Ou faut-il que le commissaire aspire à devenir un "animateur de politiques publiques de sécurité" qui consacrerait une grande part de son énergie et de son savoir faire à des procédures comme les contrats locaux de sécurité ? De tels choix sont essentiels pour l'orientation d'une gestion des compétences. Et la traduction dans les critères de mobilité des individus de tels choix en garantit la pérennité dans l'organisation. Ceci replace cette question dans une dimension, politique, d'orientation en profondeur des organisations.

\footnotetext{
${ }^{23}$ Une telle attitude qui prend acte du caractère contingent de tout argument porté et vise à le réduire en multipliant les points de vue et croisant les données, est certainement moins porteuse d'effet pervers que toute tentative de durcir le jugement autour d'une donnée jugée plus « objective », qui, une fois affichée, risque fort d'être détournée.
} 


\section{Bibliographie}

ALTER Norbert (2000), L'innovation ordinaire, Paris, Puf, 278 p. (p. 89-116). CNFPT (2001), Emplois jeunes, quel accès à l'emploi public territorial, CNFPT.

BAYSER de Anne, GEORGEAULT Valérie, MARECHAL Pierre (2004), La rémunération au mérite : mode ou nécessité ? Pour de nouveaux modes de rémunération dans les fonctions publiques d'Etat et terrioriale, Les Cahiers du groupe Bernard Brunhes, novembre, $\mathrm{n}^{\circ} 13$.

CHATZIS Konstantinos, RIBEILL Georges (2004), Le corps comme lieu d'articulation des métiers et des carrières, éclairages historiques à partir du cas des corps des ponts et chaussées et des travaux publics de 1'Etat, Rapport pour la DGAFP, Ministère de la fonction publique.

CHATZIS Konstantinos, DE CONINCK Frédéric, ZARIFIAN Philippe (1995), "L'accord ACAP 2000, la logique compétence à l'épreuve des faits", Travail et emploi, n 64, p. 35-48.

COLIN T., GRASSER B (2003), «La gestion des compétences : un infléchissement limité de la relation salariale », Travail et emploi, $\mathrm{n}^{\circ}$ 93, janvier, p. 61-73.

CONSEIL d'ETAT (2003), Rapport public 2003. Perspectives pour la fonction publique, Etudes et documents Conseil d'Etat, $\mathrm{n}^{\circ} 54$, Paris, La documentation française, $446 \mathrm{p}$.

CORTON Jean Paul (2002), "Le principe d'égalité de traitement des fonctionnaires", Actualité juridique fonction publique, mars avril, p. 4.

DREYFUS Françoise (2000), L'invention de la bureaucratie - Servir l'Etat en France, en GrandeBretagne et aux Etats-Unis (XVIIIè-XXè siècles), Paris, La Découverte - Histoire contemporaine, $287 \mathrm{p}$.

DURAN Patrice, THOENIG Jean-Claude (1996), «L'Etat et la gestion publique territoriale », ,

Revue française de sciences politiques, vol 46, $\mathrm{n}^{\circ} 4$, p. 580-622.

HOOD Christopher, LODGE Martin (2004) Competency, Bureaucracy, and Public Management Reform : A comparative Analysis, Governance, vol 17, $\mathrm{n}^{\circ}$ 3, juillet, p. 313-333.

FARNHAM David, HORTON Sylvia (2002), HRM Competency frameworks in the british Civil service, in : Horton S. et al. Ed. Competency management in the public sector, Amsterdam, IOS press, 2002, p. 33-47.

HORTON Sylvia et al. Ed. (2002). Competency management in the public sector, Amsterdam, IOS press, p. 33-47.

IRIBARNE d' Alain (2001), «Trente ans de Céreq. Des qualifications aux compétences: chronique d'un oubli accepté », Formation et Emploi, nº 76, p. 71-97.

JEANNOT Gilles (1997), «Les sources de la performance : public et privé en miroir », La Revue de l'IRES, ${ }^{\circ} 25$, automne, p. 67-87.

JEANNOT Gilles (2005), «Les métiers flous du développement rural », Sociologie du travail, 47, $1, \mathrm{p}$.

JEANNOT Gilles, PERALDI Michel (1991), L'envers des métiers: compétences politiques et pratiques professionnelles dans les directions départementales de l'Equipement, Dossiers des séminaires techniques territoires et société, $\mathrm{n}^{\circ} 15-16,244 \mathrm{p}$.

JEANNOT Gilles, LICHTENBERGER Yves (2002): "What Competency Management in France ?", in : Horton S. et al. ed. Competency management in the public sector, Amsterdam, IOS press, 2002, p. 123-134.

LOFFLER Elke, BUSSE Beate, HOPPE Ursel (2002) «Modest Beginning for Competency Management in German Public Services : Developing Competencies for Already Competent 
Lawyers », in : Horton S. et al. Ed. Competency management in the public sector, Amsterdam, IOS press, 2002, p. 105-122

MARSDEN D., RICHARDSON R., (1992), Motivation and performance related pay in the public sector, multigraf. Londonc School of Economics.

MONJARDET Dominique (1987), "Compétence et qualification comme principes d'analyse de l'action policière". De la qualification à la professionnalité, Sociologie du travail, $29, \mathrm{n}^{\circ} 1$, p. 47 58.

OBSERVATOIRE DE L'EMPLOI PUBLIC (2003), «L'approche métier au sein de la fonction publique », Rapport annuel, décembre, p. 109-116.

OCDE (1997), La rémunération à la performance pour les cadres du secteur public, Paris, Etudes hors série, $\mathrm{n}^{\circ} 15$.

PALLEZ Frédérique (1999), «Les souplesses cachées du mammouth », Le Journal de l'école de Paris, Janvier.

PARADEISE Catherine, LICHTENBERGER Yves (2001), "Compétence, Compétences », Sociologie du travail, $\mathrm{n}^{\circ} 43$, p. 33-48.

SAGLIO Jean (1987), "Les négociations de branche et l'unité du système français de relations professionnelles : le cas des négociations de classification", Droit social, n 1, p. 20-33.

SAGLIO Jean (1998), "fonctions publiques et méconnaissances sociologiques", Séminaire fonctionnaire DGAFP, 19 nov.

TROSA Sylvie (2004), « Payer les fonctionnaires au mérite ? sociétal, n 44, 2 trimestre.

TROUISSIER Jean-François (1993), «Relation d'effort et salaire au mérite », Revue française d'économie, vol VIII, n², p. 133-176.

ROZENBLATT Patrick (2000), Le mirage de la compétence, Paris, Syllepse, 267 p.

VAN VULPEN Elzeline, MOESKER franciska, «Competency-based Management in the Dutch Senior Public Service », in : Horton S. et al. (ed.) Competency management in the public sector, Amsterdam, IOS press, 2002, p. 65-76.

VELTZ Pierre (2000), Le nouveau monde industriel, Paris, Gallimard, 230 p.

ZARIFIAN Philippe (2002), "La politique de la compétence et l'appel aux connaissances à partir de la stratégie d'entreprise post-fordiste" Contribution au colloque de Nantes 13 décembre 2002.

Résumé

A partir d'une confrontation entre les principes d'une «gestion des compétences » tels qu'ils émergent progressivement dans certaines entreprises privées et administrations européennes d'une part et les modalités actuelles de gestion des carrières de deux corps de fonctionnaires (les ingénieurs des travaux publics de l'Etat et les commissaires de police), il ressort qu'une approche compétence peut correspondre aux enjeux d'une action publique co-produite sur le terrain, ne pas être forcément contre culturelle et reposer sur des instruments déjà existants. Une telle orientation suppose cependant de ne pas dissocier les dimensions techniques et comportementales d'une part et le jugement porté sur l'individu et sur les caractéristiques des postes occupés d'autre part pour offrir dans une instance paritaire des éléments d'un jugement partagé. 